\title{
The utility of ERG, CD31 and CD34 in the cytological diagnosis of angiosarcoma: an analysis of 25 cases
}

\author{
Harold C Sullivan, ${ }^{1}$ Mark A Edgar, ${ }^{1}$ Cynthia Cohen, ${ }^{1}$ Charles K Kovach, ${ }^{1}$ \\ Kim HooKim, ${ }^{2}$ Michelle D Reid ${ }^{1}$
}

${ }^{1}$ Department of Pathology and Laboratory Medicine, Emory University, Atlanta, Georgia, USA

2Department of Pathology, Louisiana State University Health Sciences Center, Shreveport, Louisiana, USA

\section{Correspondence to Dr Michelle D Reid,} Department of Pathology and Laboratory Medicine, Emory University Hospital, 1364 Clifton Road, NE, Room H189, Atlanta, GA 30322, USA: michelle.reid@emory.edu

Received 26 August 2014 Revised 3 October 2014 Accepted 9 October 2014 Published Online First 28 October 2014

\section{ABSTRACT}

Aims Erythroblast transformation specific related gene $(E R G)$, a proto-oncogene member of the erythroblast transformation specific transcription factor family, is a sensitive marker of endothelial differentiation and is expressed in vascular tumours, including angiosarcomas (AS). Immunohistochemistry is necessary for the diagnosis of AS in fine needle aspirates where low cellularity and lack of preserved tissue architecture impedes diagnosis. The aim of this study was to assess the utility of an ERG-enriched immunohistochemistry panel in the cytological diagnosis of AS.

Methods 25 AS diagnosed on fine needle aspirates were stained for ERG, CD31, CD34, and AE1/AE3. Staining intensity and percentage tumour cell positivity were evaluated. Spearman's correlation was assessed for significant correlations between antibodies.

Results Sensitivities for ERG, CD31, CD34 and AE1/ AE3 were $100 \%, 100 \%, 60 \%$ and $21 \%$, respectively. Spearman's analysis revealed that ERG and CD31 staining correlated significantly; there was no significant correlation between CD31 and CD34 staining.

Conclusions With equal sensitivity to, and strong correlation with CD31, ERG staining is highly suitable for the cytological diagnosis of AS. ERG and CD31 are more sensitive vascular markers than CD34. ERG, a nuclear stain, complements the cytoplasmic/membranous staining of CD31. Used in conjunction with CD31, ERG can corroborate the diagnosis of AS.

\section{INTRODUCTION}

Erythroblast transformation specific related gene $(E R G)$ is a proto-oncogene and a member of the erythroblast transformation specific transcription factor family whose overexpression can be seen as a result of the TMPRSS2-ERG fusion. ${ }^{1-4}$ ERG expression has been found in numerous types of cancers including prostate cancer, ${ }^{2-9}$ acute myeloid leukaemia, ${ }^{7}$ Ewing sarcoma, ${ }^{7} 10$ as well as meningiomas. ${ }^{8}$ Additionally, ERG expression has been shown to be highly expressed in endothelial cells and is implicated in the regulation of angiogenesis. ${ }^{7} 11$ 12 Consequently, ERG expression is found in various vascular tumours, benign and malignant. ${ }^{7-9}$

Angiosarcomas are rare, malignant vascular tumours that often require immunohistochemistry (IHC) to differentiate them from other sarcomas. The use of IHC is especially critical in cytological samples where only a minimal amount of tissue is present, and the absence of architecture can make diagnosis more challenging. Although studies have attempted to characterise cytomorphological features of angiosarcomas, none of the features are diagnostic. ${ }^{13-15}$ The sheer quantity of blood in samples also precludes optimal visualisation of cells present. ${ }^{14}$ Therefore, IHC and clinical history still play an important role in making the diagnosis of angiosarcoma on fine needle aspirates (FNAs).

Over the years many different immunohistochemical stains have been examined and used to demonstrate vascular differentiation in angiosarcomas. Among these stains are CD $31,{ }^{16-18}$ CD $34,{ }^{17-19}$ factor VIII, ${ }^{16-18}$ and VEGFR-3. ${ }^{16}{ }^{20}$ With increasing experience, CD31 expression has emerged as the gold standard for endothelial differentiation in angiosarcomas. ${ }^{17}$ Although CD31 and CD34 are considered markers of vascular differentiation, CD31 is not perfectly sensitive, ${ }^{21}$ while CD34 is not specific, and can also stain fibroblasts, epithelioid sarcomas (ESs), dermatofibrosarcomas, meningiomas and solitary fibrous tumours among other entities. ${ }^{21}$ Additionally some angiosarcomas, especially epithelioid angiosarcomas, coexpress endothelial and epithelial markers (ie, cytokeratins). ${ }^{22}$ The presence of 'epithelioid' cells and ambivalent IHC features make carcinoma a possible diagnostic differential, ${ }^{23-26}$ and can create a diagnostic dilemma, particularly on cytology. In such situations, the use of an additional IHC marker with high sensitivity and specificity for vascular differentiation would be especially beneficial.

Recent studies have shown the efficacy of ERG in the evaluation of vascular malignancies, including angiosarcomas. ${ }^{7-9}$ However, ERG has not been specifically assessed on cytological specimens. The aim of this study was to assess the expression and diagnostic utility of ERG in comparison with other vascular markers (CD31 and CD34), as well as a cytokeratin AE1/AE3 in cytological samples of angiosarcomas.

\section{MATERIAL AND METHODS}

\section{Tissue specimens}

A search of the pathology archives of the participating institutions revealed 25 angiosarcoma cases from 21 patients diagnosed by FNA (24 from Emory University Hospital and 1 from Louisiana State University Health Sciences Center-Shreveport) (table 1).

These included cases that were diagnosed between November 1992 and June 2013. All cytological slides were reviewed and the diagnosis was confirmed by one reference pathologist (MDR). Cases were only included in the study if sufficiently cellular paraffin-embedded cell blocks were 
Table 1 Clinicopathological parameters

\begin{tabular}{|c|c|c|c|c|c|}
\hline Case & Age (years) & Sex & Specimen & Anatomical site & Primary vs metastasis \\
\hline 1 & 2 & M & FNA & Vertebra, T7 & Records unavailable \\
\hline 2 & 2 & M & FNA & Femur, left distal & Records unavailable \\
\hline 3 & 33 & M & CT-guided FNA & Bone, sternal manubrium & Records unavailable \\
\hline 4 & 33 & M & Biopsy & Heart & Records unavailable \\
\hline 5 & 48 & M & FNA & Lung nodule, right & Metastasis \\
\hline 6 & 54 & $\mathrm{~F}$ & FNA & Subcutaneous nodule, left medial thigh & Primary \\
\hline 7 & 75 & $\mathrm{~F}$ & FNA & Subcutaneous mass, left breast & Primary \\
\hline 8 & 94 & $\mathrm{~F}$ & FNA & Skin and soft tissue, inferior aspect of right breast & Primary \\
\hline 9 & 46 & M & Pleural fluid & Pleural fluid & Primary \\
\hline 10 & 69 & $\mathrm{~F}$ & FNA & Left level 2B node & Metastasis \\
\hline 11 & 39 & M & CT-guided FNA & Soft tissues, left hemipelvis & Primary \\
\hline 12 & 76 & $\mathrm{~F}$ & FNA & Axilla, right & Recurrence \\
\hline 13 & 37 & $\mathrm{~F}$ & FNA & Liver & Records unavailable \\
\hline 14 & 63 & $\mathrm{~F}$ & FNA & Subcutaneous mass, left chest wall & Recurrence \\
\hline 15 & 50 & M & FNA & Abdominal mass, Umbilicus & Recurrence \\
\hline 16 & 47 & $\mathrm{~F}$ & CT-guided FNA & Lymph node, left inguinal & Metastasis \\
\hline 17 & 57 & $\mathrm{~F}$ & FNA & Lung Mass, Right & Metastasis \\
\hline 18 & 35 & $\mathrm{~F}$ & FNA and core & Left hemipelvis & Recurrence \\
\hline 19 & 35 & $\mathrm{~F}$ & FNA & Groin lymph node & Primary \\
\hline 20 & 64 & $M$ & FNA & Liver, left lateral segment & Primary \\
\hline 21 & 85 & $\mathrm{~F}$ & FNA & Right inguinal mass & Metastasis \\
\hline 22 & 60 & $\mathrm{~F}$ & FNA & Scalp mass & Primary \\
\hline 23 & 71 & $\mathrm{~F}$ & FNA & Right chest wall & Recurrence \\
\hline 24 & 71 & $\mathrm{~F}$ & FNA & Right chest wall & Recurrence \\
\hline 25 & 55 & $M$ & FNA & Left neck, inferior to mastoid & Metastasis \\
\hline
\end{tabular}

FNA, fine needle aspirates.

available for IHC. The diagnosis of angiosarcoma was supported by previous or subsequent surgical specimens $(n=15)$ or personal history of angiosarcoma $(n=4)$; the remaining specimens $(n=6)$ did not have available records for review. Study approval was obtained from the Emory University Investigation Review Board.

\section{Immunohistochemistry}

The 25 cases had corresponding cell blocks from which paraffin-embedded tissue sections $(5 \mu)$ were stained with ERG, CD31, CD34 and AE1/3 antibodies. For each stain a known positive case was used as a positive control with each run. Negative controls were concurrently run, with the primary antibody replaced with buffer. Staining with mouse monoclonal anti-ERG antibody to the N-terminus (BioCare Medical, Concord, California, USA) was performed with the 9FY-clone at 1:50 dilution using the Dako Autostainer (Dako, Carpinteria, California, USA). Antigen retrieval was performed using the Trilogy antigen retrieval method with EDTA at a high $\mathrm{pH}(\mathrm{pH}$ 9.0) using an electric pressure cooker for $3 \mathrm{~min}$ at 12-15 pounds per square inch $\left(120^{\circ} \mathrm{C}\right)$, and cooled for $10 \mathrm{~min}$ prior to immunostaining. The EnVision + Dual Link Kit (Dako) was the detection method, which was used according to the manufacturer's instructions, with diaminobenzidine as the chromogen, and haematoxylin as counterstain.

CD31 (Dako) staining was performed with the 7C70A clone at 1:80 dilution; CD34 (Dako) stain was performed with the QBEND 10 clone at 1:320 dilution; and AE1/3 (Dako, Carpinteria, California, USA) stain with the AE1/AE3 clone at 1:100 dilution. All three stains were carried out on the Leica Bond Max III automated system (Bond, Bannockburn, Illinois,
USA). Antigen retrieval is performed on the instrument at $\mathrm{pH}$ 6.0. The Bond Polymer Refine Detection Kit (Leica Microsystems, Bannockburn, Illinois, USA) was the detection method, which was used according to the manufacturer's instructions, with diaminobenzidine as the chromogen (brown chromogen), and haematoxylin as counterstain.

Positive ERG antigen expression was nuclear, positive CD31 and CD34 expression were cytoplasmic/membranous and AE1/ AE3 expression was cytoplasmic. Visual semiquantification was independently assessed for each stain and scored by two pathologists (MDR and MAE) for staining intensity (weak, moderate, strong) and percentage tumour cell positivity $(1: 0-25 \% ; 2: 26-$ $50 \% ; 3: 51-75 \% ; 4:>75 \%)$. Cases were considered discrepant for intensity and/or percentage positivity if there was a two-tier (or higher) difference in scoring. All discrepant cases were evaluated again and discussed between both pathologists until a consensus score was obtained. An immunostain intensity of 1 or greater (scale:0-3) in any cell was considered positive.

\section{Statistical analysis}

Distribution of gender among cases and the mean age at FNA diagnosis were determined. The number of positive staining cases, sensitivity and distribution of intensity of each stain were calculated. Additionally, correlations between antibodies were tested for significance using a Spearman's correlation.

\section{RESULTS}

The 25 patients included 15 (60\%) women and 10 men (40\%) with a mean age at diagnosis of 52 years (range 2-94 years). Tumour sites included bone $(n=3)$, skin/subcutis $(n=5)$, pleural 
fluid $(\mathrm{n}=1)$, lung $(\mathrm{n}=2)$, liver $(\mathrm{n}=2)$, heart $(\mathrm{n}=1)$, lymph nodes $(n=3)$ and soft tissue $(n=8)$.

\section{Cytological findings}

On smears malignant cells were predominantly singly dispersed; however, spindle shaped tumour cells were also present in some cases. In areas, some tumour cells showed prominent epithelioid (figure 1) as well as plasmacytoid (figure 2) features with peripherally placed nuclei and variable amounts of dense cytoplasm (figure 1). Nuclear membranes were sometimes highly irregular and convoluted. Nuclei had single, but sometimes multiple, large prominent nucleoli, some of which were elongated (bar-shaped), and variably connected to the nuclear membrane by delicate strands of chromatin (figure 1). Mitotic figures, including abnormal forms, were easily identifiable in all cases. Variably sized cytoplasmic lumina were seen on smear and cell block in several cases, and these were either empty or contained red blood cells, debris or neutrophils (figure 1). Architecturally, some cases' cells were arranged in papillary configurations (figure 3 ).

\section{Immunohistochemical results}

Sensitivity for ERG, CD31, CD34 and AE1/AE3 was $100 \%$, $100 \%, 60 \%$ and $21 \%$ respectively. In terms of staining intensity, the majority of cases stained strongly for ERG and CD31 $(76 \%$ and $72 \%$, respectively). However, the staining intensity for CD34 was much more variable with only a minority of cases staining strongly $(12 \%)$ and the remaining cases staining either moderately or weakly ( $16 \%$ and $32 \%$, respectively). Of the six cases that stained positively for AE1/AE3 most of them stained weakly, ${ }^{4}$ one stained moderately, and one stained strongly (figure 4). The number of positive cases, sensitivity and stain intensity are shown in table 2.
Spearman's analysis revealed that ERG and CD31 staining percentage correlated significantly $(p<0.0001)$, while there was no significant correlation between staining percentage of CD31 and CD34 staining $(\mathrm{p}=0.2687)$ or ERG and CD34 $(\mathrm{p}=0.5681)$ (table 3).

Additionally, staining intensity between ERG and CD31 demonstrated significant correlation $(p=0.0064)$. However, no significant correlation in staining intensity between CD31 and CD34 $(p=0.0748)$ or between ERG and CD34 $(p=0.8461)$ was observed (table 3 ).

\section{DISCUSSION}

We found that of all the vascular markers ERG had the highest sensitivity $(100 \%)$ for the detection of vascular differentiation in all 25 angiosarcomas. This finding is similar to previous reported sensitivities for ERG in the evaluation of vascular neoplasms. Miettinen et $\mathrm{al}^{7}$ examined 100 angiosarcomas among other vascular tumours and found that 96 cases showed nuclear ERG positivity, while Minner et al found ERG to be expressed in $100 \%$ of the angiosarcomas in their analysis of various tumour types. ${ }^{9}$ McKay et $a l^{27}$ also found ERG to have $100 \%$ sensitivity, as well as $100 \%$ specificity in their study of 23 cases of cutaneous angiosarcomas, supporting its utility in the evaluation of cutaneous tumours, in which CD31-positive intratumorous macrophages can potentially be a diagnostic pitfall. ${ }^{28}$ However, to date there are no large cytological studies which have evaluated and compared the performance of ERG with other vascular markers.

We found that CD31 also had 100\% sensitivity, with expression found in all 25 cases. In terms of CD34 expression, 60\% of our cases stained positively, meaning $40 \%$ of the angiosarcomas were CD34-negative. These results are consistent with what

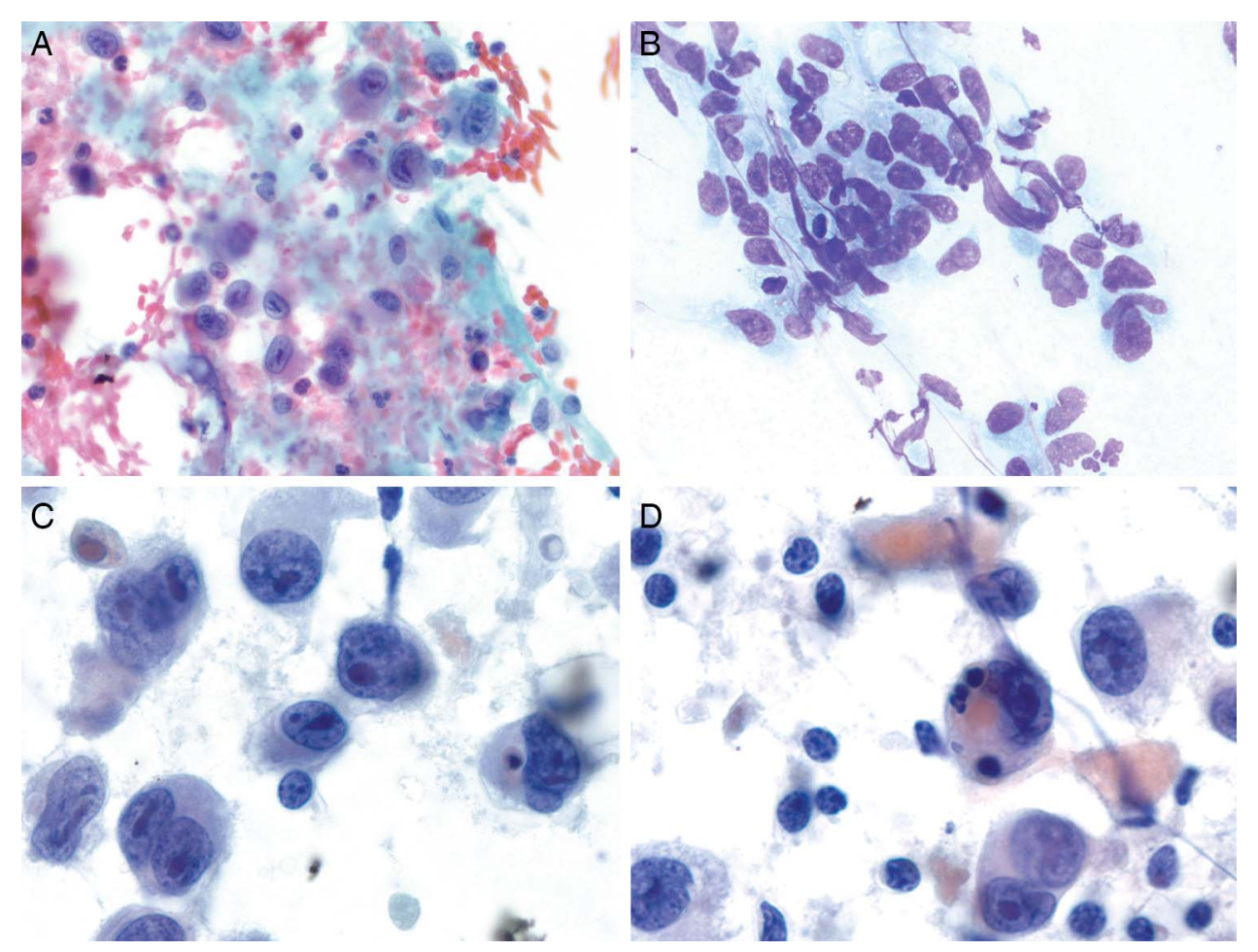

Figure 1 (A) Singly dispersed malignant epithelioid cells with abundant cytoplasm, convoluted nuclear membranes and elongated bar-shaped nucleoli (Papanicolaou stain, magnification $\times 400$ ). (B) Spindle shaped tumour nuclei with prominent nucleoli show focal peripheral cell wrapping (lower right of cluster) (Diff Quik stain, magnification $\times 400$ ). (C) Tumour cells show focal nucleolar chromatin strands that are connected to the nuclear membrane (arrow) (Papanicolaou stain, magnification $\times 1000$ ). (D) Tumour cells with cytoplasmic red blood cells and nuclear debris, consistent with haemophagocytosis (Papanicolaou stain, magnification $\times 1000$ ). 

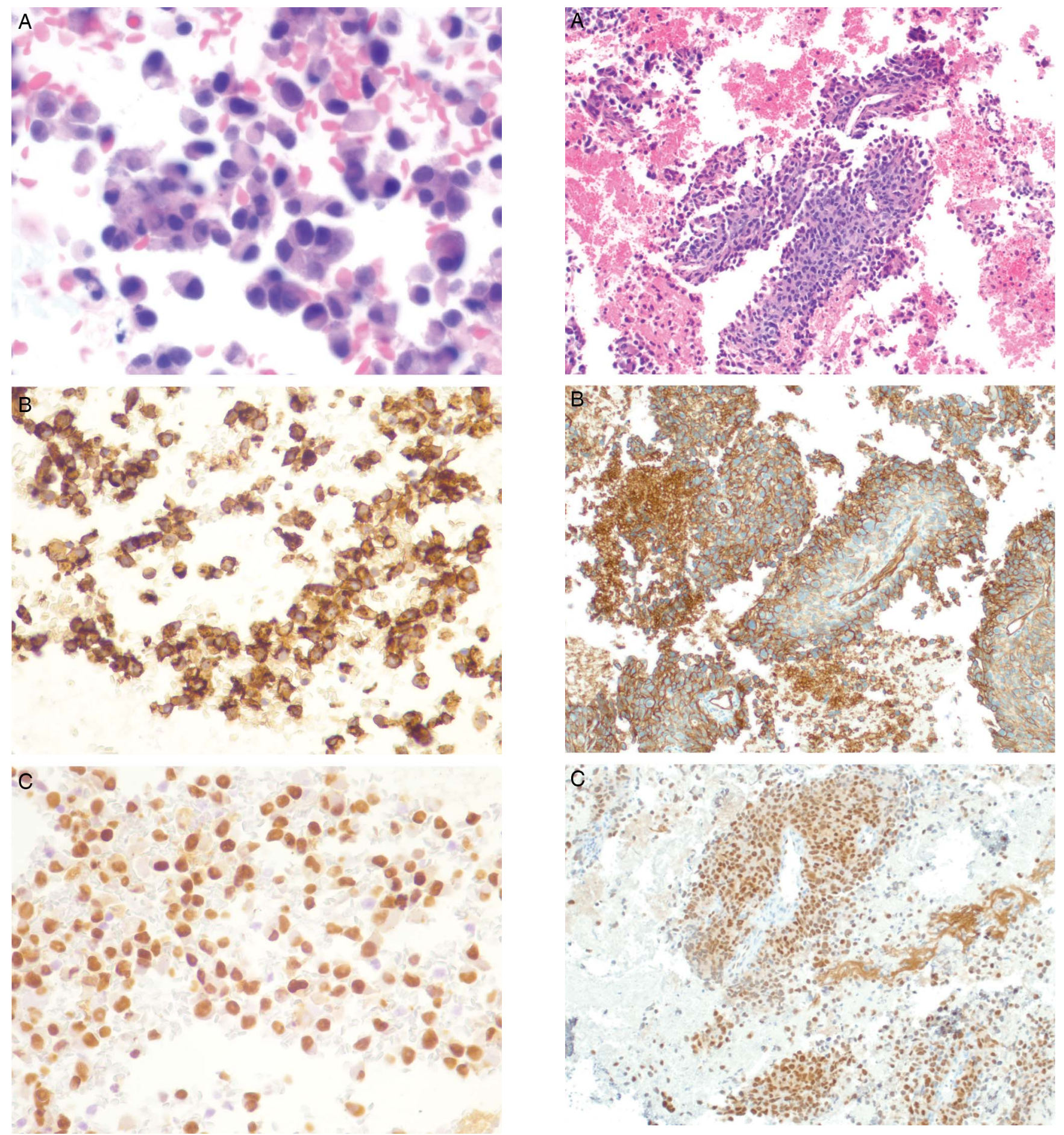

Figure 2 (A) This example of angiosarcoma shows singly dispersed malignant cells with plasmacytoid morphology on cell block (H\&E stain, magnification $\times 400$ ). Tumour cells are positive for CD31 (B) and ERG (C), and negative for CD34 (not shown), magnification $\times 400$.

Figure 3 (A) Case of angiosarcoma with papillary clusters of malignant cells with central fibrovascular cores on cell block (H\&E stain, magnification $\times 200$ ). Tumour cells are strongly positive for $C D 31$ (B) and ERG (C), magnification $\times 200$.

has been reported in the literature. According to Rao et $a l,{ }^{22}$ a tissue microarray study of 70 angiosarcomas demonstrated that $80 \%$ of cases expressed CD31 while only $63 \%$ expressed CD34. Ohsawa et al also found that CD31 had an 80\% sensitivity for angiosarcomas. ${ }^{16}$ Other studies have also shown similar trends in which CD31 appears to stain more angiosarcomas than CD34. ${ }^{17} 1829$ Taken together our study and previous studies show ERG to be comparable with, or better than, CD31

and more sensitive than $\mathrm{CD} 34$ in the detection of vascular differentiation in angiosarcomas.

When ERG was compared with CD31 and CD34, correlation analysis between ERG and CD31 staining percentage and staining intensity revealed statistical significance $(p<0.0001$ and $\mathrm{p}=0.0064$, respectively) while correlation between ERG and CD34 and between CD31 and CD34 was not statistically significant. Thus, the data suggests that ERG is just as good in the 


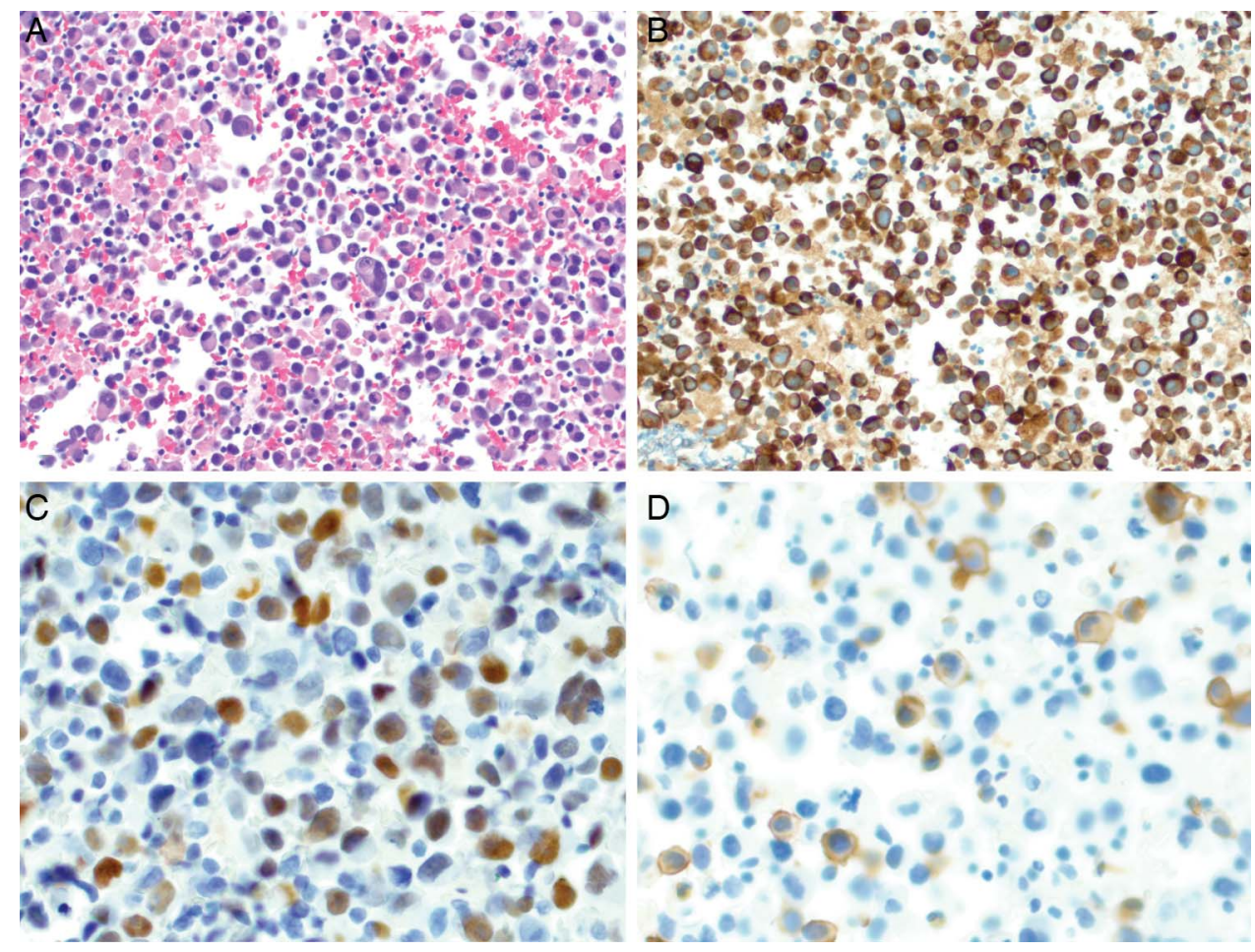

Figure 4 (A) In this example of epithelioid angiosarcoma malignant cells were singly dispersed and plasmacytoid in appearance on cell block (H\&E stain, magnification $\times 200$ ). Tumour cells are strongly and diffusely positive for AE1/AE3 (magnification $\times 200)(B)$, focally positive for ERG (C) and focally positive for CD34 (D) (magnification $\times 200$ ).

evaluation of vascular differentiation in our cohort of angiosarcomas. Furthermore, the nuclear staining pattern and the strong intensity of staining (76\% with strong intensity) of ERG helps to make stain visualisation cleaner and easier (figure 1). This is especially helpful in cytological samples where specimen preparation can lead to loss of cytoplasm and confusing background IHC staining. A crisp nuclear marker facilitates easier stain interpretation in such cases, hence the distinct advantage of ERG over CD31 and CD34 IHC.

Keratin is occasionally expressed in angiosarcomas, particularly epithelioid angiosarcomas. ${ }^{22-25} 3031$ For example, Rao et $a l^{22}$ reported keratin to be expressed in $50 \%$ of epithelioid angiosarcomas and $9 \%$ of non-epithelioid angiosarcomas. ${ }^{22}$ The coexpression of an epithelial marker is a potential diagnostic pitfall on cytological samples, especially in cases where the diagnosis of angiosarcoma is not suspected. Some angiosarcomas may appear very cohesive and epithelioid on smear and if positive for keratin can be misdiagnosed as carcinoma. In our study, cytokeratin AE1/AE3 only stained $21 \%$ of the angiosarcomas, which could have potentially been misdiagnosed as carcinomas if other vascular IHC markers had not been used. In such cases, presentation, clinical history and suspicion of vascular neoplasm can aid tremendously in the diagnosis, especially when IHC does not lead to a definitive diagnosis.

Limitations of our study include the small sample size $(n=25)$ as well as limitation of sample type, which only included angiosarcomas. We did attempt to include other malignant vascular neoplasms, however cytology specimens of these entities were limited. In fact in the study period (November 1992 to June 2013), only one Kaposi's sarcoma and two epithelioid haemangioendothelioma FNAs with corresponding cell blocks were available. Given the low yield, the cases were not included in the analysis. Additionally, the absence of non-vascular neoplasms also precluded the calculation of specificity, as there were no true negative cases in the cohort.

In terms of ERG-positivity, the differential diagnosis is short. Beyond endothelial tissue, the only carcinoma consistently shown to express ERG is prostatic adenocarcinoma. ${ }^{6-8}{ }^{32}$ In the evaluation of 1129 carcinomas, Liu et $a l^{32}$ found that ERG expression was only observed in prostatic adenocarcinoma. Furthermore, the cytomorphological features of prostate adenocarcinoma are distinct from those of angiosarcomas, lacking the intracytoplasmic lumina, intracellular red blood cells, wellformed vessels and spindled and epithelioid cells described in

Table 2 Sensitivity and staining intensity

\begin{tabular}{llllll}
\hline Stain & $\begin{array}{l}\text { Positive cases no. } \\
\text { (Sensitivity \%) }\end{array}$ & $\begin{array}{l}\text { Negative } \\
\text { cases no. (\%) }\end{array}$ & $\begin{array}{l}\text { Cases with strong } \\
\text { intensity staining no. (\%) }\end{array}$ & $\begin{array}{l}\text { Cases with moderate } \\
\text { intensity staining no. (\%) }\end{array}$ & $\begin{array}{l}\text { Cases with weak } \\
\text { intensity staining no. (\%) }\end{array}$ \\
\hline ERG & $25(100)$ & $0(0)$ & $19(76)$ & $5(20)$ & $1(4)$ \\
CD31 & $25(100)$ & $0(0)$ & $18(72)$ & $6(24)$ & $1(4)$ \\
CD34 & $15(60)$ & $10(40)$ & $3(12)$ & $8(32)$ & $4(16)$ \\
AE1/AE3 & $5(21)$ & $19(79)$ & $1(4)$ & $1(4)$ & $4(17)$ \\
\hline
\end{tabular}

ERG, erythroblast transformation specific (ETS)-related gene; No., number. 
Table 3 Spearman's correlation

\begin{tabular}{llcr}
\hline Stains & Variable & Spearman's coefficient & p Value \\
\hline CD31 vs ERG & \% staining & 0.7039 & $<0.0001$ \\
& Intensity & 0.5303 & 0.0064 \\
CD31 vs CD34 & \% staining & 0.2300 & 0.2687 \\
& Intensity & 0.3627 & 0.0748 \\
ERG vs CD34 & \% staining & 0.1199 & 0.5681 \\
& Intensity & -0.0409 & 0.8461 \\
\hline
\end{tabular}

ERG, erythroblast transformation specific (ETS)-related gene.

the literature. ${ }^{13}{ }^{14}$ Although ERG stains a fraction of Ewing's sarcoma ${ }^{10}$ and acute myeloid leukaemias, ${ }^{7}$ these morphologically are composed of small round blue cells and larger blast cells, both of which also have a distinct immunophenotypical profile from angiosarcomas beyond ERG positivity.

An additional diagnostic pitfall is ES, which could mistakenly be interpreted as angiosarcoma on FNA, as ES can be CD34-positive as well as ERG-positive. Miettinen et al found that although negative for the ERG rearrangements by fluorescence in situ hybridization, 38\% of 109 ESs were positive for ERG by IHC. However, none of the cases were positive for CD31. ${ }^{33}$ However, recently loss of INI1/SMARCB1 expression has been shown to be of clinical utility in identifying ES; ${ }^{33-35}$ for example, Miettinen $e a^{33}$ found that INI1/SMARCB1 staining was absent in all ES but only one epithelioid angiosarcoma. Thus, the importance of using a panel of stains cannot be underestimated when evaluating these soft tissue tumours. Another study compared two ERG antibodies, one directed towards the $\mathrm{N}$-terminus and the other towards the C-terminus, in the evaluation of ESs. The results demonstrated that the $\mathrm{N}$-terminus antibody stained 19 of 28 cases while the C-terminus antibody only detected 1 of 29 cases; ${ }^{36}$ this study highlights the importance of antibody clone selection when excluding angiosarcoma. We used the N-terminal ERG antibody in our study.

It is also important to bear in mind that ERG stains a variety of benign and malignant neoplasms. Thus, not all vascular lesions that stain with ERG are angiosarcomas. Along with the 25 angiosarcomas, we concurrently stained 12 haemangiomas, all of which stained positive with ERG (data not shown). Therefore, when evaluating a suspected vascular lesion on FNA specimens, it is vital to note the cytomorphology and

\section{Take home messages}

- Erythroblast transformation specific related gene (ERG) is a sensitive marker of endothelial differentiation and is expressed in vascular tumours, including angiosarcomas (AS).

- ERG IHC was expressed in all 25 cytologic cases of AS (i.e. $100 \%$ sensitivity).

- ERG IHC has equal sensitivity to, and strong correlation with CD31.

- ERG and CD31 are more sensitive vascular markers than CD34.

- ERG, a nuclear stain, complements the cytoplasmicl membranous staining of CD31.

- Used in conjunction with CD31, ERG can corroborate the diagnosis of AS. differentiate benign cellular features from malignant ones. Furthermore, looking for some of the aforementioned cytological features of angiosarcomas, such as atypical epithelioid and spindled cells along with intracytoplasmic vacuoles with and without erythrocytes, can help guide the pathologist.

With equal sensitivity to and strong correlation with CD31, ERG staining is highly suitable for the cytological diagnosis of angiosarcoma. ERG and CD31 are more sensitive vascular markers than CD34. Also, ERG is a nuclear stain, which complements the cytoplasmic/membranous staining of CD31. Thus, when used in conjunction with CD31, ERG can corroborate the diagnosis of angiosarcoma.

Acknowledgements The authors thank Diane Lawson, Jennifer Jiang and Sonal Bhimji-Pattni, Immunohistochemistry Laboratory, Emory University Hospital, for their contributions and excellent work.

Contributors All the authors contributed to the idea, conception and contents of the document. HCS and MDR wrote the first drafts of the document; the remaining authors reviewed and/or amended these drafts. CKK performed the statistical analysis.

\section{Competing interests None.}

Provenance and peer review Not commissioned; externally peer reviewed.

\section{REFERENCES}

1 Kumar-Sinha C, Tomlins SA, Chinnaiyan AM. Recurrent gene fusions in prostate cancer. Nat Rev Cancer 2008;8:497-511.

2 Chaux A, Albadine R, Toubaji A, et al. Immunohistochemistry for ERG expression as a surrogate for TMPRSS2-ERG fusion detection in prostatic adenocarcinomas. Am J Surg Pathol 2011;35:1014-20.

3 Falzarano SM, Zhou M, Carver $P$, et al. ERG gene rearrangement status in prostate cancer detected by immunohistochemistry. Virchows Arch 2011;459:441-7.

4 Park K, Tomlins SA, Mudaliar KM, et al. Antibody-based detection of ERG rearrangement-positive prostate cancer. Neoplasia 2010;12:590-8.

5 van Leenders GJ, Boormans JL, Vissers CJ, et al. Antibody EPR3864 is specific for ERG genomic fusions in prostate cancer: implications for pathological practice. Mod Pathol 2011;24:1128-38.

6 Verdu M, Trias I, Roman R, et al. ERG expression and prostatic adenocarcinoma. Virchows Arch 2013;462:639-44.

7 Miettinen M, Wang ZF, Paetau A, et al. ERG transcription factor as an immunohistochemical marker for vascular endothelial tumors and prostatic carcinoma. Am J Surg Pathol 2011;35:432-41.

8 Yaskiv 0 , Rubin BP, He $\mathrm{H}$, et al. ERG protein expression in human tumors detected with a rabbit monoclonal antibody. Am J Clin Pathol 2012;138:803-10.

9 Minner S, Luebke AM, Kluth $M$, et al. High level of Ets-related gene expression has high specificity for prostate cancer: a tissue microarray study of 11483 cancers. Histopathology 2012:61:445-53.

10 Wang WL, Patel NR, Caragea $M$, et al. Expression of ERG, an Ets family transcription factor, identifies ERG-rearranged Ewing sarcoma. Mod Pathol 2012;25:1378-83.

11 Birdsey GM, Dryden NH, Amsellem V, et al. Transcription factor Erg regulates angiogenesis and endothelial apoptosis through VE-cadherin. Blood 2008; 111:3498-506.

12 Birdsey GM, Dryden NH, Shah AV, et al. The transcription factor Erg regulates expression of histone deacetylase 6 and multiple pathways involved in endothelial cell migration and angiogenesis. Blood 2012;119:894-903.

13 Boucher LD, Swanson PE, Stanley MW, et al. Cytology of angiosarcoma. Findings in fourteen fine-needle aspiration biopsy specimens and one pleural fluid specimen. Am J Clin Pathol 2000;114:210-19.

14 Minimo C, Zakowski M, Lin O. Cytologic findings of malignant vascular neoplasms: a study of twenty-four cases. Diagn Cytopathol 2002;26:349-55.

15 Liu K, Layfield LJ. Cytomorphologic features of angiosarcoma on fine needle aspiration biopsy. Acta Cytol 1999;43:407-15.

16 Ohsawa M, Naka N, Tomita Y, et al. Use of immunohistochemical procedures in diagnosing angiosarcoma. Evaluation of 98 cases. Cancer 1995;75:2867-74.

17 Poblet E, Gonzalez-Palacios F, Jimenez FJ. Different immunoreactivity of endothelial markers in well and poorly differentiated areas of angiosarcomas. Virchows Arch 1996:428:217-21.

18 Yang XJ, Zheng JW, Zhou Q, et al. Angiosarcomas of the head and neck: a clinico-immunohistochemical study of 8 consecutive patients. Int J Oral Maxillofac Surg 2010;39:568-72.

19 Traweek ST, Kandalaft PL, Mehta P, et al. The human hematopoietic progenitor cell antigen (CD34) in vascular neoplasia. Am J Clin Pathol 1991;96:25-31. 


\section{Original article}

20 Folpe AL, Veikkola T, Valtola R, et al. Vascular endothelial growth factor receptor-3 (VEGFR-3): a marker of vascular tumors with presumed lymphatic differentiation, including Kaposi's sarcoma, kaposiform and Dabska-type hemangioendotheliomas, and a subset of angiosarcomas. Mod Pathol 2000;13:180-5.

21 Miettinen $M$, Lindenmayer AE, Chaubal A. Endothelial cell markers CD31, CD34, and $\mathrm{BNH} 9$ antibody to $\mathrm{H}$ - and $\mathrm{Y}$-antigens--evaluation of their specificity and sensitivity in the diagnosis of vascular tumors and comparison with von Willebrand factor. Mod Pathol 1994;7:82-90.

22 Rao $\mathrm{P}$, Lahat $\mathrm{G}$, Arnold $\mathrm{C}$, et al. Angiosarcoma: a tissue microarray study with diagnostic implications. Am J Dermatopathol 2013;35:432-7.

23 Meis-Kindblom JM, Kindblom LG. Angiosarcoma of soft tissue: a study of 80 cases. Am J Surg Pathol 1998;22:683-97.

24 Gagner JP, Yim JH, Yang GC. Fine-needle aspiration cytology of epithelioid angiosarcoma: a diagnostic dilemma. Diagn Cytopathol 2005;33:429-33.

25 Fletcher $C D$, Beham A, Bekir $S$, et al. Epithelioid angiosarcoma of deep soft tissue: a distinctive tumor readily mistaken for an epithelial neoplasm. Am J Surg Pathol 1991;15:915-24.

26 Siddaraju N, Soundararaghavan J, Bundele MM, et al. Fine needle aspiration cytology of epithelioid angiosarcoma: a case report. Acta Cytol 2008;52:109-13.

27 McKay KM, Doyle LA, Lazar AJ, et al. Expression of ERG, an Ets family transcription factor, distinguishes cutaneous angiosarcoma from histological mimics. Histopathology 2012;61:989-91.
28 McKenney JK, Weiss SW, Folpe AL. CD31 expression in intratumoral macrophages: a potential diagnostic pitfall. Am J Surg Pathol 2001;25:1167-73.

29 Kuzu I, Bicknell R, Harris AL, et al. Heterogeneity of vascular endothelial cells with relevance to diagnosis of vascular tumours. I Clin Pathol 1992;45:143-8.

30 Wenig BM, Abbondanzo SL, Heffess CS. Epithelioid angiosarcoma of the adrenal glands. A clinicopathologic study of nine cases with a discussion of the implications of finding "epithelial-specific" markers. Am I Surg Pathol 1994; 18:62-73.

31 Gray MH, Rosenberg AE, Dickersin GR, et al. Cytokeratin expression in epithelioid vascular neoplasms. Hum Pathol 1990;21:212-17.

32 Liu $\mathrm{H}$, Shi J, Wilkerson $\mathrm{M}$, et al. Immunohistochemical evaluation of ERG expression in various benign and malignant tissues. Ann Clin Lab Sci 2013;43:3-9.

33 Miettinen M, Wang Z, Sarlomo-Rikala M, et al. ERG expression in epithelioid sarcoma: a diagnostic pitfall. Am J Surg Pathol 2013;37:1580-5.

34 Frank R, Sadri N, Bhatti T, et al. Proximal-type Epithelioid Sarcoma of the Head and Neck (HN): A Study with Immunohistochemical and Molecular Analysis of SMARCB1. J Clin Exp Oncol 2013;2. pii: 1000106.

35 Rekhi B, Jambhekar NA. Immunohistochemical validation of INI1/SMARCB1 in a spectrum of musculoskeletal tumors: an experience at a Tertiary Cancer Referral Centre. Pathol Res Pract 2013;209:758-66.

36 Stockman DL, Hornick JL, Deavers MT, et al. ERG and FLI1 protein expression in epithelioid sarcoma. Mod Pathol 2013;27:496-501. 